\title{
Epidemiology and Antifungal Resistance in Invasive Candidiasis
}

\author{
A. C. Rodloff, D. Koch, R. Schaumann
}

Institute for Medical Microbiology and Epidemiology of Infectious Diseases, University Hospital of Leipzig, Leipzig, Germany

\begin{abstract}
The epidemiology of Candida infections has changed over the last two decades: The number of patients suffering from such infections has increased dramatically and the Candida species involved have become more numerous as Candida albicans is replaced as an infecting agent by various non-C. albicans species (NAC). At the same time, additional antifungal agents have become available. The different Candida species may vary in their susceptibility for these various antifungals. This draws more attention to in vitro susceptibility testing. Unfortunately, several different test methods exist that may deliver different results. Moreover, clinical breakpoints (CBP) that classify test results into susceptible, intermediate and resistant are controversial between CLSI and EUCAST. Therefore, clinicians should be aware that interpretations may vary with the test system being followed by the microbiological laboratory. Thus, knowledge of actual MIC values and pharmacokinetic properties of individual antifungal agents is important in delivering appropriate therapy to patients
\end{abstract}

\section{INTRODUCTION}

In 2001, McNeil et al. reported that (in the USA) "from 1980 through 1997, the annual number of deaths in which an invasive mycosis was listed on the death certificate (multiple-cause mortality) increased from 1557 to 6534" (Table 1, Fig. 1) [1]. Augmentation of fungal infections had been published earlier i.e. by Beck-Sague and Jarvis [2] and Edmond et al. [3] for the USA and by Lamagni et al. [4] for England and Wales (Fig. 2). There are several possible reasons for this change. An important one might be the increase in lifespan in the populations of the developed world and the age related loss of immune-competence. An increase of systemic fungal infections is probably also due to more intensive treatment schemes for hematological and oncological patients causing prolonged neutropenic phases. Finally, more effective antibacterial treatments allow patients with infections to survive longer without necessarily overcoming the underlying diseases and thus leaving them susceptible to other opportunistic infections. Eggimann et al. [5] summarized prior surgery, acute renal failure, previous yeast colonization, neutropenia, antibacterial therapy, parenteral nutrition, and central venous catheters as risk factors for invasive Candidia infections.

\section{Change in Epidemiology}

The increase in incidence of Candida infections barely preceded the introduction of fluconazole in 1990. This azole agent combined good activity against Candida albicans with reduced toxicity as compared to i.e. polyene anti-fungals. It is orally and parentally available and has a reliable that means linear pharmacokinetic profile [6], which makes it easy to handle. Not surprisingly, fluconazole became the agent of choice for many fungal infections as well as for prophylactic purposes, at that time often being applied in rather low doses. Although there is inconclusive evidence, many experts in the field believe that it was the selective pressure exerted by this therapeutic concept that caused changes in the epidemiology [7]. While in earlier years, $C$. albicans was responsible most of the invasive fungal infections (Table 2) [8], gradually more and more non-C. albicans species (NAC) were found as offending agents (Table 3) [9-16]. While the data show similar tendencies in the prevalence of various Candi$d a$ ssp. worldwide, considerable differences can be observed as well. However, these do not lend themselves to further interpretation since significant differences in the demographics of the patients observed seem to be obvious. This change in prevalence of various Candida spp. is nevertheless of clinical importance, since individual species vary in their susceptibility to various antifungal agents. While national and international surveillance is important to recognize trends in epidemiology it is, however, of utmost importance to gain knowledge about the local epidemiology as this information should guide the empiric therapy of patients.

\section{SusceptibILITY TESTING}

Some of the already cited and many other studies have also reported on the in-vitro susceptibility of Candida spp. For various reasons it is difficult to assess the various results. There are currently several test methods for performing these assays. Standardized methods have been published by the Clinical and Laboratory Standards Institute (CLSI) of the USA $[17,18]$ and by the European Committee on Antimicrobial Susceptibility Testing (EUCAST) [19]. The two broth dilution methods are not identical as methodological differences include glucose concentration, inoculum size, shape of microtitration wells (flat or round), and end-point reading (visual or spectrophotometric). However, it appears 
Table 1. Ranking of underlying causes of deaths due to infectious diseases in the United States in 1980 and 1997 [1].

\begin{tabular}{llrlll}
\hline & \multicolumn{2}{c}{1980} & & \multicolumn{2}{c}{1997} \\
\cline { 5 - 6 } Rank & Type of infection & No. of deaths & & Type of infection & No. of deaths \\
\hline 1 & Respiratory tract & 56,966 & & Respiratory tract & 87,181 \\
2 & Septicemia & 9,438 & & Septicemia & 22,396 \\
3 & Kidney/UTI & 8,006 & & HIV/AIDS & 16,524 \\
4 & Heart & 2,486 & & Kidney/UTI & 13,413 \\
5 & Tuberculosis & 2,333 & & Heart & 5,577 \\
6 & Bacterial meningitis & 1,402 & & Hepatobiliary & 4,596 \\
7 & Gastrointestinal & 1,377 & & Mycoses & 2,370 \\
8 & Hepatobiliary & 1,277 & & Tuberculosis & 1,259 \\
9 & Perinatal & 1,035 & & Gastrointestinal & 1,053 \\
10 & Mycoses & 828 & & Perinatal & 820 \\
\hline
\end{tabular}

Note: Categories of infectious diseases identified by anatomic site rather than by causative microorganism did not have any microorganism specified in the death-certificate data. UTI = urinary tract infedtion.

Table 2. Species distribution of Candida from cases of invasive candidiasis ${ }^{\text {[ }}[8]$.

\begin{tabular}{|c|c|c|c|c|c|c|}
\hline \multirow{2}{*}{ Species } & \multicolumn{6}{|c|}{$\%$ of total cases $b$} \\
\hline & 1997-1998 & 1999 & 2000 & 2001 & 2002 & 2003 \\
\hline C. albicans & 73.3 & 69.8 & 68.1 & 65.4 & 61.4 & 62.3 \\
\hline C. glabrata & 11.0 & 9.7 & 9.5 & 11.1 & 10.7 & 12.0 \\
\hline C. tropicalis & 4.6 & 5.3 & 7.2 & 7.5 & 7.4 & 7.5 \\
\hline C. parapsilosis & 4.2 & 4.9 & 5.6 & 6.9 & 6.6 & 7.3 \\
\hline C. krusei & 1.7 & 2.2 & 3.2 & 2.5 & 2.6 & 2.7 \\
\hline C. guilliermondii & 0.5 & 0.8 & 0.8 & 0.7 & 1.0 & 0.8 \\
\hline C. Iusitaniae & 0.5 & 0.5 & 0.5 & 0.6 & 0.5 & 0.6 \\
\hline C. Kefyr & 0.2 & 0.4 & 0.5 & 0.4 & 0.4 & 0.5 \\
\hline C. rugosa & 0.03 & 0.03 & 0.2 & 0.7 & 0.6 & 0.4 \\
\hline C. famata & 0.08 & 0.2 & 0.5 & 0.2 & 0.4 & 0.3 \\
\hline C. inconspicua & & & 0.08 & 0.1 & 0.2 & 0.3 \\
\hline C. novegensis & & & 0.08 & 0.1 & 0.07 & 0.1 \\
\hline C. dubliniensis & & & 0.01 & 0.08 & 0.1 & 0.05 \\
\hline C. lipolytica & & & 0.06 & 0.06 & 0.06 & 0.08 \\
\hline C. zeylanoides & & & 0.03 & 0.08 & 0.02 & 0.04 \\
\hline C. pelliculosa & & & & 0.06 & 0.05 & 0.04 \\
\hline Canida spp. & 3.9 & 6.0 & 3.7 & 3.3 & 7.9 & 4.9 \\
\hline Total no. of cases & 22,533 & 20,998 & 11,698 & 21,804 & 24,680 & 33,002 \\
\hline
\end{tabular}

a Data compiled from the ARTEMIS DISK Surveillance Program, 1997 to 2003 (221).

b Includes all specimen types and all hospitals from a total of 127 different institutions in 39 countries.

${ }^{\mathrm{c}}$ Candida species not otherwise identified.

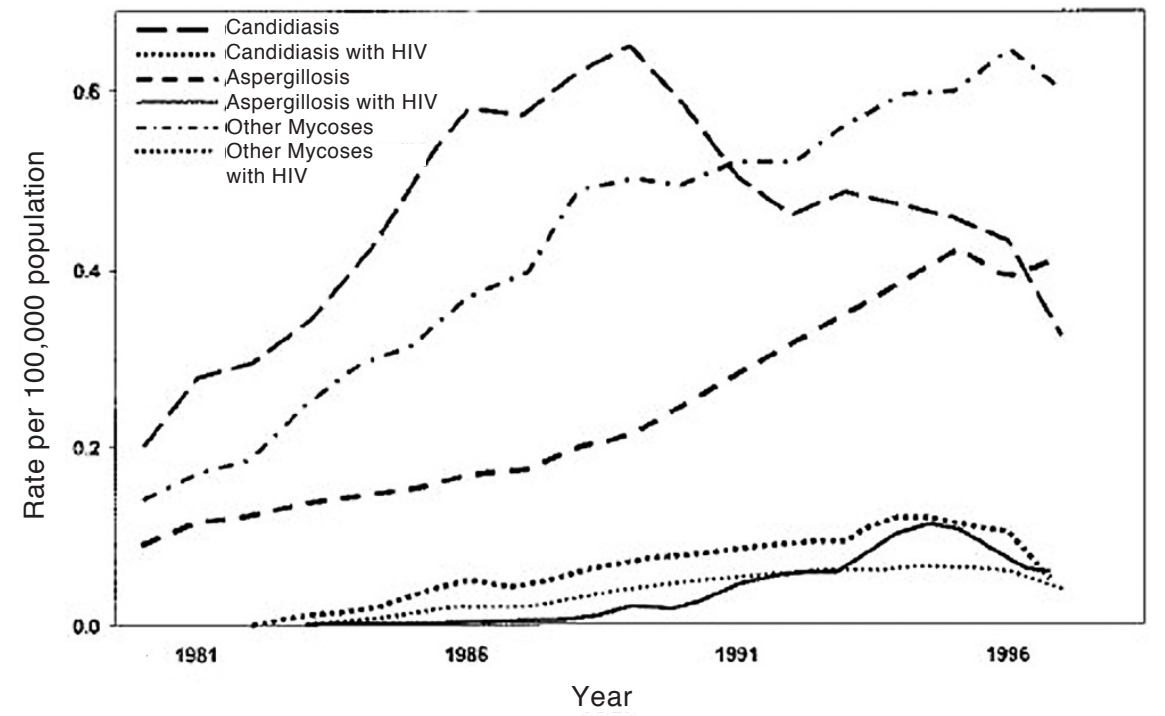

Fig. 1. Mortality in the United States, 1980-1997, due to candidiasis, and other mycoses in persons infected and persons not infected with HIV [1]. 
Table 3. Species distribution of Candida in blood stream infections in various studies.

\begin{tabular}{|c|c|c|c|c|c|c|c|c|}
\hline Reference & 9 Artemis & 10 Sentry & 11 Horn & 12 Ostrosky & 13 Cisterna & 14 Arendrup & 15 Fleck & 16 Borg \\
\hline Year & $2005-2007$ & 2008-2009 & 2004-2008 & 1995/1999 & 2008-2009 & 2004-2009 & 2004-2006 & 2004-2005 \\
\hline Location & worldwide & worldwide & USA & USA & Spain & Denmark & Germany & Germany \\
\hline $\mathrm{n}$ & 88647 & 1354 & 2019 & 2000 & 984 & 2901 & 512 & 561 \\
\hline C. albicans & 65 & 48.4 & 45.6 & 36.7 & 49.1 & 57.1 & 43 & 58.5 \\
\hline C. glabrata & 11.7 & 18.2 & 26 & 22.9 & 13.6 & 21.1 & 31.3 & 19.1 \\
\hline C. tropicalis & 8 & 10.6 & 8.1 & 15.4 & 10.8 & 4.8 & 11.7 & 7.5 \\
\hline C. parapsilosis & 5.6 & 17.1 & 15.6 & 19.6 & 20.7 & 3.7 & 5.7 & 8 \\
\hline C. krusei & 2.5 & 2 & 2.5 & 2.5 & 2.1 & 4.1 & 3.7 & 1.4 \\
\hline C. guilliermondii & 0.6 & & 0.3 & & & & & 1.1 \\
\hline C. Insitaniae & 0.6 & & 0.8 & 1 & & & $\leq 1$ & 0.2 \\
\hline C. kefyr & 0.6 & & & & & & $\leq 1$ & \\
\hline C. inconspicua & 0.3 & & & & & & $\leq 1$ & 1.1 \\
\hline C. famata & 0.3 & & & & & & $\leq 1$ & 0.7 \\
\hline C. rugosa & 0.2 & & & & & & & 0.2 \\
\hline C. dubliniensis & 0.2 & & 0.4 & 0.9 & & 2.6 & & 1.1 \\
\hline C. norvegensis & 0.1 & & & & & & & 0.4 \\
\hline C. lipolytica & 0.06 & & & & & & $\leq 1$ & \\
\hline C. sake & 0.08 & & & & & & $\leq 1$ & \\
\hline C. pelliculosa & 0.05 & & & & & & & \\
\hline C. apicola & 0.06 & & & & & & & \\
\hline C. zeylanoides & 0.02 & & & & & & & \\
\hline C. valida & 0.01 & & & & & & $\leq 1$ & \\
\hline C. intermedia & 0.01 & & & & & & $\leq 1$ & 0.4 \\
\hline C. pulcherrima & $<0.01$ & & & & & & & \\
\hline C. haemulonii & $<0.01$ & & & & & & & \\
\hline C. stellatoidea & $<0.01$ & & & & & & & \\
\hline C. utilis & $<0.01$ & & & & & & $\leq 1$ & 0.4 \\
\hline C. bumicola & $<0.01$ & & & & & & & \\
\hline C. lambica & $<0.01$ & & & & & & & \\
\hline C. ciferrii & $<0.01$ & & & & & & & \\
\hline C. colliculosa & $<0.01$ & & & & & & $\leq 1$ & 0.4 \\
\hline C. bolmii & $<0.01$ & & & & & & & \\
\hline C. marina & $<0.01$ & & & & & & & \\
\hline C. sphaerica & $<0.01$ & & & & & & & \\
\hline Candida spp. & 4 & & 0.7 & & 3.6 & 5.1 & 4.7 & \\
\hline
\end{tabular}
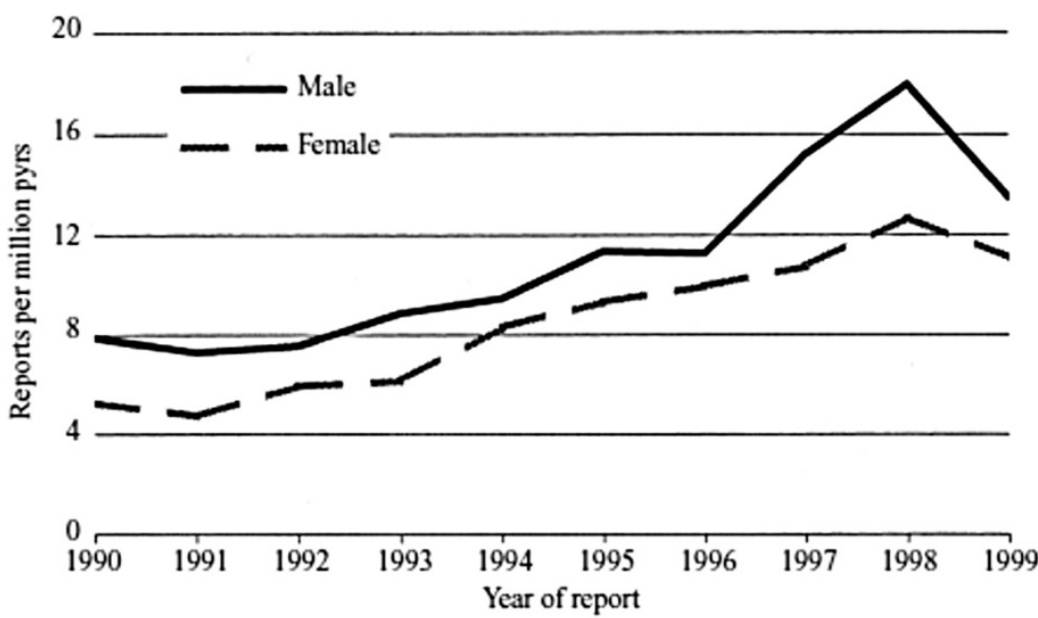

Fig. 2. Annual rates of candidosis laboratory reports, by sex (England and Wales: 1990-9) [4].

that they result in similar MIC levels for polyenes, azoles and echinocandins for identical isolates with a few noted exceptions [20-23]. This is especially true if isolates with defined resistance mechanisms are being tested [22, 23]. However, some "drug/bug" combinations seem to offer particular test problems i.e. Caspofungin and C. glabrata [22]. Moreover, EUCAST (and for that matter Etest) results have a tendency for one to two dilution steps lower MIC values [20, 23]. There are also some commercially available test devices that have been tested in their performance. A high degree of correlation with the reference methods was found for the Etest by various authors [22, 23]. The percentage of strains classified as resistant in vitro by the EUCAST procedure and as susceptible in vitro by the VITEK 2 system was $2.6 \%$, and as resistant by the CLSI method and as susceptible by the VITEK 2 was $1.6 \%$ (very major error) [24]. The difference observed for the Vitek 2 
Table 4. In vitro susceptibilities of Candida spp. to fluconazole and voriconazole as determined by CLSI disk diffusion testing ${ }^{\mathrm{a}}$ [9].

\begin{tabular}{|c|c|c|c|c|c|c|}
\hline \multirow[b]{2}{*}{ Species } & \multicolumn{3}{|c|}{ Fluconazole ${ }^{b}$} & \multicolumn{3}{|c|}{ Voriconazole $^{\mathrm{b}}$} \\
\hline & No. of isolates & $\% \mathrm{~S}$ & $\% \mathrm{R}$ & No. of isolates & $\% \mathrm{~S}$ & $\% \mathrm{R}$ \\
\hline C. albicans & 128,625 & 98.0 & 1.4 & 125,965 & 98,5 & 1.2 \\
\hline C. glabrata & 23,305 & 68.7 & 15.7 & 22,968 & 82.9 & 10.0 \\
\hline C. tropicalis & 15,546 & 91.0 & 4.1 & 15,198 & 89.5 & 5.4 \\
\hline C. parapsilosis & 12,788 & 93.2 & 3.6 & 12,453 & 97.0 & 1.8 \\
\hline C. kerusei & 5,079 & 8.6 & 78.3 & 5,005 & 83.2 & 7.6 \\
\hline C. guilliermondii & 1,410 & 73.5 & 11.4 & 1,375 & 90.5 & 5.7 \\
\hline C. Iusitaniae & 1,233 & 92.1 & 5.4 & 1,215 & 96.7 & 2.0 \\
\hline C. kefyr & 1,044 & 96.5 & 2.7 & 1,032 & 98.7 & 0.9 \\
\hline C. inconspicua & 566 & 22.6 & 53.2 & 563 & 90.6 & 3.9 \\
\hline C. famata & 622 & 79.1 & 10.3 & 606 & 90.3 & 5.0 \\
\hline C. rugosa & 603 & 49.9 & 41.8 & 580 & 69.3 & 21.2 \\
\hline C. dubliniensis & 310 & 96.1 & 2.6 & 308 & 98.4 & 1.0 \\
\hline C. norvegensis & 248 & 41.9 & 40.7 & 247 & 91.5 & 4.0 \\
\hline C. lipolytica & 130 & 66.2 & 28.5 & 128 & 77.3 & 14.1 \\
\hline C. sake & 87 & 85.1 & 11.5 & 87 & 92.0 & 6.9 \\
\hline C. pelliculosa & 87 & 89.7 & 6.9 & 86 & 94.2 & 4.7 \\
\hline C. apicola & 57 & 98.2 & 1.8 & 57 & 98.2 & 1.8 \\
\hline C. zeylanoides & 70 & 67.1 & 24.3 & 67 & 85.1 & 6.0 \\
\hline C. valida & 21 & 23.8 & 61.9 & 22 & 81.8 & 13.6 \\
\hline C. intermedia & 24 & 95.8 & 4.2 & 25 & 100.0 & 0.0 \\
\hline C. pulcherrima & 14 & 100.0 & 0.0 & 14 & 100.0 & 0.0 \\
\hline C. haemulonii & 9 & 88.9 & 11.1 & 9 & 88.9 & 11.1 \\
\hline C. stellatoidea & 7 & 85.7 & 0.0 & 7 & 85.7 & 14.3 \\
\hline C. utilis & 6 & 83.3 & 0.0 & 7 & 100.0 & 0.0 \\
\hline C. bumicola & 6 & 50.0 & 50.0 & 6 & 50.0 & 33.3 \\
\hline C. lambica & 5 & 0.0 & 80.0 & 5 & 40.0 & 20 \\
\hline C. ciferrii & 2 & 50.0 & 50.0 & 2 & 50.0 & 0.0 \\
\hline C. colliculosa & 2 & 100.0 & 0.0 & 2 & 100.0 & 0.0 \\
\hline C. holmii & 1 & 100.0 & 0.0 & 1 & 100.0 & 0.0 \\
\hline C. marina & 1 & 0.0 & 0.0 & 1 & 100.0 & 0.0 \\
\hline C. sphaerica & 1 & 100.0 & 0.0 & 1 & 100.0 & 0.0 \\
\hline Candida spp. & 9,744 & 86.2 & 8.9 & 9,577 & 93.6 & 4.1 \\
\hline
\end{tabular}

a Isolates were obtained from 133 istitutions, 2001 tp 2007.

${ }^{\mathrm{b}}$ Fluconazole and voriconazole disk diffusion testing was performed in accordance with CLSI document M44-A (7). The interpretive breakpoint (zone diameters) were as follows: $\mathrm{S}, \geq 19 \mathrm{~mm}$ (fluconazole) and $\geq 17 \mathrm{~min}$ (voriconazole); $\mathrm{R}, \leq 14 \mathrm{~mm}$ (fluconazole) and $\leq 13 \mathrm{~mm}$ (voriconazole).

${ }^{\mathrm{c}}$ Candida species, not otherwise specified.

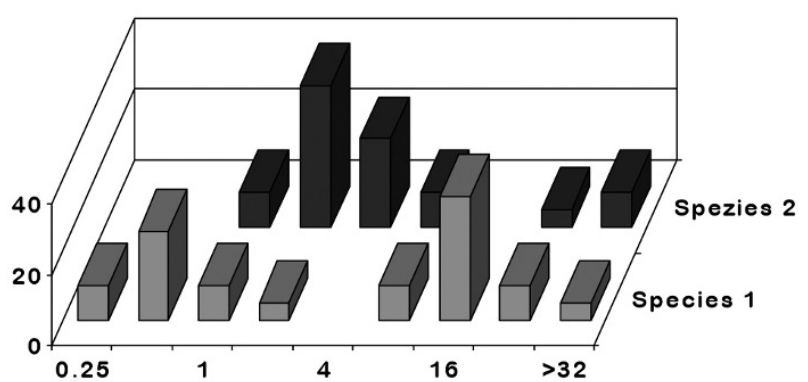

Fig. 3. Two very different fictitious MIC distributions resulting in the same MIC50/90 values: 2 and $32 \mathrm{mg} / \mathrm{L}$

results and CLSI and EUCAST is driven by the fact that there are differences in clinical breakpoints (CBP) suggested by the two organizations. However these results indicate that although the CBP of EUCAST and CLSI are significantly different, currently only few strains are affected by this difference.

CBP are used to classify MIC results into susceptible (S), intermediate (I) (CLSI for some strange reason "susceptible dose dependent"; S-DD), and resistant (R), respectively. This has complicated to assess and compare studies were only percentages of S, I and R are published. The same is true for published MIC50/90 values, as very different MIC distributions might be behind these numbers. To illustrate this further, two very different fictitious distributions resulting in the same MIC50/90 values are given with Figure 3. Therefore, meaningful surveillance data should be published as MIC distributions. That allows for evaluation of the results even at a later date when i.e. CBP had been changed as has happened in the past. To circumvent the problem with differences in CBP published by various organizations, it has been suggested to use epidemiological cut -off values to distinguish between wild type (W'T) organisms without any resistance mechanisms and non-wild type (N-WT) strains with higher MIC values that are thought or known to possess a resistance mechanism [22, 25]. This would put in vitro test results on the safe side as long as the particular species is a target for the antifungal agent in question. Obviously, CBP should not divide WT distributions as this will cause arbitrary test results. Monitoring the development of N-WT strains in surveillance studies allows one to analyze the spread of resistance mechanisms. 
Table 5. In vitro susceptibilities of 5,346 clinical isolates of Candida spp. to anidulafungin, caspofingin, and micafungin [38].

\begin{tabular}{|c|c|c|c|c|c|c|c|c|c|c|c|c|c|}
\hline \multirow[t]{2}{*}{ Organism } & \multirow{2}{*}{$\begin{array}{l}\text { o. of isloates } \\
\text { tested }\end{array}$} & \multirow{2}{*}{$\begin{array}{c}\text { Antifungal } \\
\text { agent }\end{array}$} & \multicolumn{11}{|c|}{ Cumulative $\%$ of isolates susceptible at a MIC $(\mu \mathrm{g} / \mathrm{ml})$ of ${ }^{\text {a }}$} \\
\hline & & & 0.007 & 0.015 & 0.03 & 0.06 & 0.12 & 0.25 & 0.5 & 1 & 2 & 4 & $\geq 8$ \\
\hline \multirow[t]{3}{*}{ C. albicans } & 2,869 & Anidulafungin & 6.2 & 33.5 & 69.5 & 92.4 & 99.1 & 99.5 & 99.5 & 99.6 & 100.0 & & \\
\hline & & Caspofungin & 1.7 & 26.7 & 74.2 & 97.1 & 99.3 & 99.9 & 100.0 & & & & \\
\hline & & Micafungin & 11.9 & 80.6 & 96.4 & 99.3 & 99.4 & 99.5 & 99.6 & 100.0 & & & \\
\hline \multirow[t]{3}{*}{ C. parapsilosis } & 759 & Anidulafungin & & & 0.3 & 0.3 & 0.3 & 1.4 & 4.7 & 27.9 & 92.5 & 100.0 & \\
\hline & & Caspofungin & & 0.1 & 0.5 & 3.3 & 10.7 & 52.2 & 89.5 & 98.6 & 99.9 & 100.0 & \\
\hline & & Micafungin & & 0.1 & 0.3 & 0.3 & 0.5 & 6.1 & 24.4 & 79.3 & 100.0 & & \\
\hline \multirow[t]{3}{*}{ C. glabrata } & 747 & Anidulafungin & & 0.4 & 7.8 & 62.4 & 93.6 & 99.4 & 99.7 & 99.9 & 99.9 & 100.0 & \\
\hline & & Caspofungin & & 7.0 & 65.2 & 95.3 & 98.4 & 99.2 & 99.7 & 99.9 & 99.9 & 99.9 & 100.0 \\
\hline & & Micafungin & 13.7 & 91.4 & 97.9 & 98.9 & 99.5 & 99.9 & 99.9 & 100.0 & & & \\
\hline \multirow[t]{3}{*}{ C. tropicalis } & 625 & Anidulafungin & 3.2 & 24.2 & 75.7 & 95.0 & 98.4 & 99.4 & 99.5 & 99.5 & 100.0 & & \\
\hline & & Caspofungin & 1.3 & 31.0 & 79.7 & 97.3 & 99.0 & 99.7 & 99.7 & 99.8 & 99.8 & 99.8 & 100.0 \\
\hline & & Micafungin & 4.0 & 39.5 & 77.6 & 96.3 & 98.6 & 99.5 & 99.7 & 100.0 & & & \\
\hline \multirow[t]{3}{*}{ C. krusei } & 136 & Anidulafungin & & 2.9 & 47.1 & 90.4 & 99.3 & 99.3 & 100.0 & & & & \\
\hline & & Caspofungin & & 0.7 & 0.7 & 41.9 & 75.7 & 94.9 & 99.3 & 100.0 & & & \\
\hline & & Micafungin & & 2.2 & 13.2 & 85.3 & 96.3 & 100.0 & & & & & \\
\hline \multirow[t]{3}{*}{ C. guilliermondii } & 61 & Anidulafungin & & & & & 3.3 & 6.6 & 13.1 & 57.4 & 90.2 & 100.0 & \\
\hline & & Caspofungin & & & 1.6 & 4.9 & 11.5 & 39.3 & 80.3 & 95.1 & 95.1 & 95.1 & 100.0 \\
\hline & & Micafungin & & 3.3 & 3.3 & 6.6 & 11.5 & 21.3 & 65.6 & 98.4 & 100.0 & & \\
\hline \multirow[t]{3}{*}{ C. lusitaniae } & 58 & Anidulafungin & & & & 1.7 & 13.8 & 43.1 & 96.6 & 100.0 & & & \\
\hline & & Caspofungin & & & 3.4 & 6.9 & 44.8 & 89.7 & 96.6 & 100.0 & & & \\
\hline & & Micafungin & & & 1.7 & 8.6 & 63.8 & 96.6 & 98.3 & 100.0 & & & \\
\hline \multirow[t]{3}{*}{ C. kefyr } & 37 & Anidulafungin & & 2.7 & 10.8 & 56.8 & 100.0 & & & & & & \\
\hline & & Caspofungin & 13.5 & 97.3 & 100.0 & & & & & & & & \\
\hline & & Micafungin & & 5.4 & 40.5 & 100.0 & & & & & & & \\
\hline \multirow[t]{3}{*}{ C. famata } & 24 & Anidulafungin & & 4.2 & 16.7 & 20.8 & 20.8 & 20.8 & 25.0 & 50.0 & 100.0 & & \\
\hline & & Caspofungin & & 4.2 & 12.5 & 20.8 & 37.5 & 70.8 & 70.8 & 95.8 & 100.0 & & \\
\hline & & Micafungin & & 4.2 & 16.7 & 16.7 & 20.8 & 33.3 & 75.0 & 91.7 & 100.0 & & \\
\hline \multirow[t]{3}{*}{ Candida spp. } & 30 & Anidulafungin & 3.3 & 30.0 & 50.0 & 63.3 & 63.3 & 73.3 & 86.7 & 93.3 & 96.7 & 96.7 & 100.0 \\
\hline & & Caspofungin & & 16.7 & 43.3 & 63.3 & 73.3 & 96.7 & 100.0 & & & & \\
\hline & & Micafungin & & 20.0 & 53.3 & 66.7 & 66.7 & 86.7 & 100.0 & & & & \\
\hline \multirow[t]{3}{*}{ Total } & 5,246 & Anidulafungin & 3.7 & 21.1 & 48.9 & 72.6 & 82.0 & 83.4 & 84.7 & 88.7 & 98.8 & 99.9 & 100.0 \\
\hline & & Caspofungin & 1.2 & 19.7 & 59.4 & 79.6 & 84.0 & 91.9 & 98.1 & 99.7 & 99.9 & 99.9 & 100.0 \\
\hline & & Micafungin & 8.8 & 60.9 & 75.6 & 81.3 & 83.3 & 85.0 & 88.5 & 97.0 & 100.0 & & \\
\hline
\end{tabular}

a Values corresponding to MICs at which at least $90 \%$ of isolates are inhibited are listed in bold types.

Table 6. In vitro antifungal agent susceptibilities of Candida and Cryptococcus isolates collected by the SENTRY Program in 2006 to 2007 [39].

\begin{tabular}{|c|c|c|c|c|c|}
\hline \multirow{2}{*}{$\begin{array}{l}\text { Species (no. of isolates) } \\
\text { and drug }\end{array}$} & \multirow{2}{*}{$\mathrm{MIC}_{50} / \mathrm{MIC}_{90}(\mu \mathrm{g} / \mathrm{ml})$} & \multirow{2}{*}{ MIC range $(\mu \mathrm{g} / \mathrm{ml})$} & \multicolumn{3}{|c|}{$\%$ by category ${ }^{a}$} \\
\hline & & & S & SDD & $\mathrm{R}^{\mathrm{b}}$ \\
\hline \multicolumn{6}{|l|}{ C. parapsilosis (238) } \\
\hline Anidulafungin & $2 / 2$ & $0.03-4$ & 95.4 & - & 4.6 \\
\hline Caspofungin & $0.5 / 1$ & $0.06-4$ & 99.6 & - & 0.4 \\
\hline Amphotericin B & $1 / 1$ & $0.25-1$ & 99.6 & - & 0.4 \\
\hline $5-\mathrm{FC}$ & $\leq 0.5 / \leq 0.5$ & $\leq 0.5->64$ & 98.7 & $(0.0)$ & 1.3 \\
\hline Fluconazole & $1 / 4$ & $\leq 0.5-32$ & 96.6 & 3.4 & 0.0 \\
\hline Itraconazole & $0.25 / 0.25$ & $\leq 0.015-2$ & 40.8 & 57.1 & 2.1 \\
\hline Posaconazole & $0.12 / 0.25$ & $\leq 0.06-1$ & - & - & - \\
\hline Voriconazole & $\leq 0.06 / 0.12$ & $\leq 0.06-2$ & 99.6 & 0.4 & 0.0 \\
\hline
\end{tabular}

\section{Resistance Mechanisms}

A number of different resistance mechanisms have been described in Candida spp. Often, several of these mechanisms are combined to result in a stepwise development of clinically relevant resistance. Resistance to i.e. fluconazole can be caused i.e. by alterations in sterol biosynthesis, by mutations in the drug target enzyme, sterol $14 \alpha$-demethylase, which lowers its affinity for fluconazole, by increased expression of the ERG11 gene encoding for this enzyme, or by overexpression of genes coding for membrane transport proteins of the $\mathrm{ABC}$ transporter (CDR1/CDR2) or the major facilitator (MDR1) superfamilies [26].

Similarly Candida isolates were found with reduced susceptibility to echinochandins that showed mutations in selected regions of $f k s 1$, the gene encoding the echinocandin target enzyme 1-3-b-D-glucan synthase [27]. In particular, mutations of the serine at position 645 and also, in some cases, at position 641 
Table 7. Antifungal susceptibilities of rare Candida bloodstream isolates [41].

\begin{tabular}{|c|c|c|c|c|c|c|c|c|c|c|c|c|c|c|c|c|c|}
\hline \multirow{2}{*}{ Species } & \multirow{2}{*}{$\begin{array}{l}\text { No. of } \\
\text { isolates }\end{array}$} & \multirow{2}{*}{ Antifungal agent } & \multicolumn{15}{|c|}{ No. inhibited at MIC $(\mu \mathrm{g} / \mathrm{ml})$ of: } \\
\hline & & & 0.007 & 0.015 & 0.03 & 0.06 & 0.12 & 0.25 & 0.5 & 1 & 2 & 4 & 8 & $16^{b}$ & 32 & $64^{c}$ & $\geq 128$ \\
\hline \multirow[t]{7}{*}{ C. lusitaniae } & 171 & Amphotericin ${ }^{a}$ & & & & 3 & 18 & 74 & 66 & 7 & 1 & 0 & 1 & 1 & & & \\
\hline & 171 & Fluconazole & & & & & 20 & 52 & 64 & 20 & 5 & 3 & 1 & 1 & 3 & 2 & \\
\hline & 171 & Posaconazole & 2 & 25 & 63 & 58 & 15 & 4 & 1 & 3 & & & & & & & \\
\hline & 171 & Voriconazole & 123 & 33 & 3 & 4 & 2 & 3 & 2 & 1 & & & & & & & \\
\hline & 96 & Anidulafungin & & & & 5 & 13 & 36 & 40 & 2 & & & & & & & \\
\hline & 166 & Caspofungin & & 1 & 4 & 6 & 68 & 66 & 17 & 3 & 0 & 1 & & & & & \\
\hline & 80 & Micafungin & 1 & 0 & 4 & 8 & 44 & 21 & 1 & 1 & & & & & & & \\
\hline \multirow[t]{7}{*}{ C. guilliermondii } & 174 & Amphotericin & 1 & 0 & 1 & 8 & 63 & 62 & 24 & 6 & 2 & 1 & 1 & 0 & 0 & 5 & \\
\hline & 175 & Fluconazole & & & & & & 1 & 4 & 4 & 69 & 68 & 13 & 7 & 2 & 3 & 4 \\
\hline & 175 & Posaconazole & & 1 & 9 & 10 & 44 & 73 & 25 & 3 & 4 & $\mathbf{0}$ & 0 & 6 & & & \\
\hline & 175 & Voriconazole & 2 & 11 & 19 & 83 & 43 & 8 & 1 & 0 & 2 & 0 & 0 & 6 & & & \\
\hline & 107 & Anidulafungin & & 1 & & 2 & 6 & 5 & 7 & 37 & 41 & 8 & & & & & \\
\hline & 156 & Caspofungin & & & 2 & 9 & 21 & 33 & 58 & 21 & 4 & 2 & 2 & 4 & & & \\
\hline & 96 & Micafungin & & 3 & 1 & 4 & 10 & 13 & 33 & 27 & 4 & 0 & 0 & 1 & & & \\
\hline \multirow[t]{7}{*}{ C. orthopsilosis } & 102 & Amphotericin & & & & & & 7 & 29 & 35 & 23 & 8 & & & & & \\
\hline & 102 & Fluconazole & & & & & & 6 & 28 & 45 & 9 & 8 & 4 & 1 & 0 & 1 & \\
\hline & 102 & Posaconazole & & 1 & 12 & 40 & 30 & 8 & 11 & & & & & & & & \\
\hline & 102 & Voriconazole & 1 & 24 & 40 & 24 & 1 & 10 & 1 & 1 & & & & & & & \\
\hline & 52 & Anidulafungin & & & & & & 3 & 12 & 28 & 9 & & & & & & \\
\hline & 91 & Caspofungin & 1 & 0 & 3 & 17 & 37 & 25 & 8 & & & & & & & & \\
\hline & 51 & Micafungin & & & & & 2 & 25 & 21 & 3 & & & & & & & \\
\hline \multirow[t]{7}{*}{ C. keffyr } & 74 & Amphotericin & & & & & & & 20 & 43 & 10 & 0 & 0 & 0 & 0 & 1 & \\
\hline & 74 & Fluconazole & & & & & 11 & 44 & 12 & 6 & 1 & & & & & & \\
\hline & 74 & Posaconazole & 1 & 3 & 17 & 21 & 23 & 8 & 1 & & & & & & & & \\
\hline & 74 & Voriconazole & 50 & 18 & 4 & 2 & & & & & & & & & & & \\
\hline & 58 & Anidulafungin & & 1 & 5 & 31 & 21 & & & & & & & & & & \\
\hline & 74 & Caspofungin & 11 & 56 & 6 & 1 & & & & & & & & & & & \\
\hline & 53 & Micafungin & & 4 & 22 & 26 & 1 & & & & & & & & & & \\
\hline \multirow[t]{7}{*}{ C. pelliculosa } & 40 & Amphotericin & & & & & 3 & 14 & 21 & 2 & & & & & & & \\
\hline & 40 & Fluconazole & & & & & & & & 2 & 7 & 24 & 7 & & & & \\
\hline & 40 & Posaconazole & & & & 1 & 1 & 6 & 4 & 14 & 12 & 2 & & & & & \\
\hline & 40 & Voriconazole & & 1 & 1 & 1 & 21 & 13 & 3 & & & & & & & & \\
\hline & 14 & Anidulafungin & 2 & 9 & 2 & 1 & & & & & & & & & & & \\
\hline & 37 & Caspofungin & 1 & 16 & 17 & 3 & & & & & & & & & & & \\
\hline & 14 & Micafungin & & 5 & 7 & 2 & & & & & & & & & & & \\
\hline \multirow[t]{7}{*}{ C. famata } & 16 & Amphotericin & & & & & 1 & 6 & 8 & 0 & 0 & 1 & & & & & \\
\hline & 16 & Fluconazole & & & & & & & & 1 & 5 & 6 & 1 & 3 & & & \\
\hline & 16 & Posaconazole & & 2 & 0 & 0 & 1 & 5 & 7 & 1 & 2 & & & & & & \\
\hline & 16 & Voriconazole & & & 2 & 5 & 4 & 3 & 0 & 1 & 1 & & & & & & \\
\hline & 16 & Anidulafungin & & & & 2 & 0 & 0 & 0 & 5 & 9 & & & & & & \\
\hline & 16 & Caspofungin & & & 1 & 2 & 2 & 5 & 3 & 2 & 1 & & & & & & \\
\hline & 16 & Micafungin & & & 1 & 1 & 0 & 2 & 5 & 5 & 2 & & & & & & \\
\hline C. metapsilosis & 30 & Amphotericin & & & & & 1 & 5 & 12 & 9 & 2 & 1 & & & & & \\
\hline & 30 & Fluconazole & & & & & & 1 & 0 & 19 & 9 & 1 & & & & & \\
\hline & 30 & Posaconazole & & 1 & 7 & 15 & 5 & 1 & 0 & 1 & & & & & & & \\
\hline & 30 & Voriconazole & 1 & 4 & 22 & 2 & 1 & & & & & & & & & & \\
\hline & 11 & Anidulafungin & & & & & & 5 & 3 & 2 & 1 & & & & & & \\
\hline & 24 & Caspofungin & & & 1 & 5 & 14 & 3 & 0 & 1 & & & & & & & \\
\hline & 11 & Micafungin & & & & & & 7 & 3 & 1 & & & & & & & \\
\hline C. dubliniensis & 18 & Amphotericin & & & & 1 & 8 & 7 & 1 & 1 & & & & & & & \\
\hline & 18 & Fluconazole & & & & & 8 & 9 & 0 & 0 & 0 & 0 & 1 & & & & \\
\hline & 18 & Posaconazole & & 4 & 6 & 7 & 1 & & & & & & & & & & \\
\hline & 18 & Voriconazole & 11 & 5 & 2 & & & & & & & & & & & & \\
\hline & 11 & Anidulafungin & & & 7 & 2 & 0 & 2 & & & & & & & & & \\
\hline & 17 & Caspofungin & & 2 & 6 & 9 & & & & & & & & & & & \\
\hline & 9 & Micafungin & & 4 & 3 & 2 & & & & & & & & & & & \\
\hline C. lipolytica & 16 & Amphotericin & & & & & & & 1 & 5 & 5 & 4 & 1 & & & & \\
\hline & 16 & Fluconazole & & & & & & & 1 & 1 & 6 & 6 & 1 & 0 & 0 & 1 & \\
\hline
\end{tabular}

Continued on following page

have been associated with decreased susceptibility to echinochandins [28].

It should be noted that there is at least for fluconazole a clear relation between MIC values, pharmacokinetics (expressed in serum AUC) and outcome (Fig. 4) [29]. Since resistance development against i.e. echino- candines so far is very limited, it is obviously very difficult to establish such a correlation. This problem is further aggravated by the fact that current antifungals still leave much to be desired as they i.e. do not reach the cure rates of antibacterial agents for susceptible bacteria. Moreover, the described resistance mecha- 
Table 7 continued

\begin{tabular}{|c|c|c|c|c|c|c|c|c|c|c|c|c|c|c|c|c|c|}
\hline \multirow{2}{*}{ Species } & \multirow{2}{*}{$\begin{array}{l}\text { No. of } \\
\text { isolates }\end{array}$} & \multirow{2}{*}{ Antifungal agent } & \multicolumn{15}{|c|}{ No. inhibited at MIC $(\mu \mathrm{g} / \mathrm{ml})$ of: } \\
\hline & & & 0.007 & 0.015 & 0.03 & 0.06 & 0.12 & 0.25 & 0.5 & 1 & 2 & 4 & 8 & $16^{b}$ & 32 & $64^{c}$ & $\geq 128$ \\
\hline \multirow{12}{*}{ C. rugosa } & 16 & Posaconazole & & & & & 2 & 1 & 8 & 4 & 0 & 1 & & & & & \\
\hline & 16 & Voriconazole & & 1 & 5 & 7 & 2 & 0 & 0 & 1 & & & & & & & \\
\hline & 10 & Anidulafungin & & & & & & 3 & 4 & 2 & 1 & & & & & & \\
\hline & 15 & Caspofungin & & & & & 6 & 9 & & & & & & & & & \\
\hline & 10 & Micafungin & & & & & & 6 & 3 & 1 & & & & & & & \\
\hline & 16 & Amphotericin & & & & & & & 4 & 3 & 7 & 1 & 0 & 0 & 0 & 1 & \\
\hline & 16 & Fluconazole & & & & & & & 1 & 0 & 4 & 3 & 2 & 4 & 1 & 1 & \\
\hline & 16 & Posaconazole & & & & 6 & 2 & 5 & 3 & & & & & & & & \\
\hline & 16 & Voriconazole & 2 & 1 & 4 & 3 & 1 & 3 & 2 & & & & & & & & \\
\hline & 16 & Anidulafungin & & & & 3 & 1 & 4 & 3 & 3 & 0 & 0 & 2 & & & & \\
\hline & 16 & Caspofungin & & & 1 & 1 & 0 & 1 & 2 & 8 & 0 & 1 & 0 & 2 & & & \\
\hline & 16 & Micafungin & & & 1 & 3 & 3 & 5 & 2 & 0 & 0 & 0 & 0 & 2 & & & \\
\hline
\end{tabular}

${ }^{a}$ Amphotericin B MICs were determined by Etest.

${ }^{b}$ For posaconazole, voriconazole, anidulafungin, caspofungin, and micafungin, isolates for which MICs are reported to be $16 \mu \mathrm{g} / \mathrm{ml}$ encompass all isolates for which MICs were $>8 \mu \mathrm{g} / \mathrm{ml}$.

${ }^{c}$ For amphotericin B, isolates for which the MIC is reported to be $64 \mu \mathrm{g} / \mathrm{ml}$ encompass all isolates for which MICs were $>32 \mu \mathrm{g} / \mathrm{ml}$.

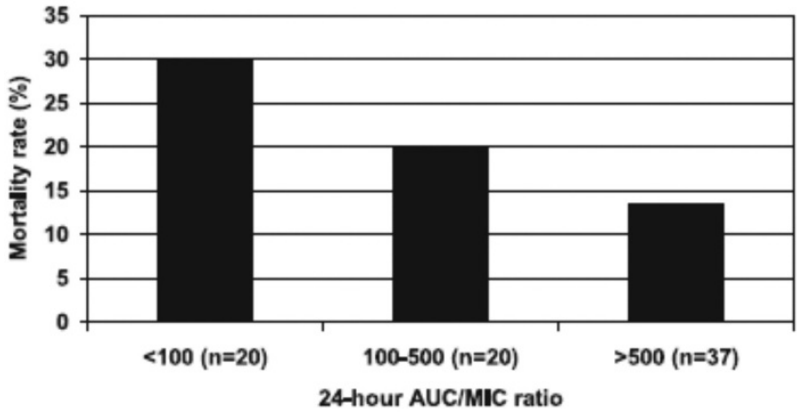

Fig. 4. Mortality rate stratified by tertiles and fluconazole AUC/MIC at $24 \mathrm{~h}(\mathrm{P}=0.09$ using logistic regression controlling for time to initiation of fluconazole therapy) [29].

nisms were at least in individual cases associated with clinical failure of the involved patients [30-35]. This has raised concern over the CBP as suggested by the CLSI. For a discussion of this subject see [36, 37].

\section{RESistanCE SuRVEILLANCE}

Among the worldwide largest and long run surveillance systems is the ARTEMIS program. In a recent publication [9], comparative susceptibility data for fluconazole and voriconazole for more than 190,000 isolates collected from 2001 to 2007 were provided and analysis of resistance rates by year, geographic location, hospital location, and specimen type for selected species were included. The data were collected employing the CLSI disk diffusion method and are summarized in Table 4. They show that fluconazole resistance has to be expected especially in C. glabrata, krusei, guillermondii, famata, inconspicua, rugosa, norvegensis and some other rarer species. There seem to be some but no complete crossresistance with voriconazole which leaves the latter as an option in appropriate cases. A trend toward increased resistance over the most recent 3 years (2005 to 2007) was observed for voriconazole and some species with low prevalence such as C. famata (1.1\% to 5.7\%), C. norvegensis $(0.0 \%$ to $6.9 \%)$, C. lipolytica $(0.0 \%$ to $11.1 \%$ ), and C. pelliculosa (14.3\% to $16.7 \%)$. However, there was no trend toward increased resistance to voriconazole among the fluconazole-resistant species
C. glabrata, C. krusei, C. guilliermondii, C. rugosa, and C. inconspicua.

MIC distributions for echinocandins have been published by Pfaller et al. (Table 5) [39]. The results of this study demonstrate the comparable spectrum and potency of all three available echinocandin antifungals against a large collection of clinically important $C$ andi$d a$ spp. It also highlights the fact that species such as C. parapsilosis and C. guilliermondii exhibit decreased susceptibilities to all three echinocandins. The clinical relevance of these elevated MICs currently remains doubtful.

The data collected by the SENTRY surveillance program were published as MIC50/90 values and interpreted results according to CLSI, only [39, 40]. However, this study included most important antifungals. An excerpt for C. parapsilosis is given with Table 6. German data were published in the same way [15], not offering significant differences to the SENTRY results with the exception of a high degree of flucytosine resistance in C. krusei and C. tropicalis. Finally, susceptibility data for rare Candida isolates have been collected by Diekema et al. [41] (Table 7) and Chen et al. [42] that might help to guide therapy in these cases.

\section{CONCLUSION}

Meanwhile in vitro methods are available to assess reliably the susceptibility of fungal isolates. There are, however, considerable differences in the evaluation of the results, as CLSI and EUCAST breakpoints vary. If isolates with known resistance mechanisms that have been shown to be clinically relevant at least in individual cases shall not be categorized as susceptible, some CLSI CBP need to be reconsidered. Despite the fact that a number of new antifungals are nowadays available, clinical results of antifungal therapy leave much to be desired. Hence, optimization of empiric therapy according to the local epidemiological situation and reevaluation of the therapeutic regimen when susceptibility results become available should carefully be followed. With our expanded knowledge on pharmacokinetics of antifungal compounds, MIC data could be valuable at least when treating invasive fungal infections. More information about MICs of clinical iso- 
lates and outcome of the particular patients would be helpful to establish further and validate current CBP.

\section{REFERENCES}

1. Michael M. McNeil, Stephanie L. Nash, Rana A. Hajjeh, Maureen A. Phelan, Laura A. Conn, Brian D. Plikaytis, and David W. Warnock. 2001. Trends in Mortality Due to Invasive Mycotic Diseases in the United States, 1980-1997. Clin Infect Dis 33(5): 641-647

2. Beck-Sague, C. and W. R. Jarvis. 1993. Secular trends in the epidemiology of nosocomial fungal infections in the United States, 1980-1990. National Nosocomial Infections Surveillance System. J Infect Dis 167:1247-1251.

3. Edmond, M. B., Wallace, S. E., McClish, D. K., Pfaller, M. A., Jones, R. N. \& Wenzel, R. P. (1999). Nosocomial bloodstream infections in United States hospitals: a threeyear analysis. Clin Infect Dis 29, 239-244.

4. Lamagni TL, Evans BG, Shigematsu M, Johnson EM. 2001. Emerging trends in the epidemiology of invasive mycoses in England and Wales (1990-9). Epidemiol Infect 126(3):397-414.

5. Eggimann, P., Garbino, J. \& Pittet, D. 2003. Epidemiology of Candida species infections in critically ill non-immunosuppressed patients. Lancet Infect Dis 3, 685-702.

6. Washton H. 1989. Review of fluconazole: a new triazole antifungal agent. Diagn Microbiol Infect Dis 12(4 Suppl): 229S-233S.

7. Olivier Leroy, MD; Jean-Pierre Gangneux, MD, PhD; Philippe Montravers, MD, PhD;Jean-Paul Mira, MD, PhD; Franc, ois Gouin, MD; Jean-Pierre Sollet, MD; Jean Carlet, $\mathrm{MD}$; Jacques Reynes, MD, PhD; Michel Rosenheim, MD; Bernard Regnier, MD, PhD; Olivier Lortholary, MD, PhD; for the AmarCand Study Group. 2009. Epidemiology, management, and risk factors for death of invasive Candida infections in critical care: A multicenter, prospective, observational study in France (2005-2006). Crit Care Med 37 (5), 1612-18

8. M. A. Pfaller and D. J. Diekema. 2007. Epidemiology of Invasive Candidiasis: a Persistent Public Health Problem. Clin Microbiol Rev 20 (1): 133-163

9. M. A. Pfaller, D. J. Diekema, D. L. Gibbs, V. A. Newell, D. Ellis, V. Tullio, A. Rodloff, W. Fu, T. A. Ling, and the Global Antifungal Surveillance Group. 2010. Results from the ARTEMIS DISK Global Antifungal Surveillance Study, 1997 to 2007: a 10.5-Year Analysis of Susceptibilities of Candida Species to Fluconazole and Voriconazole as Determined by CLSI Standardized Disk Diffusion. J Clin Microbiol. 48 (4) 1366-1377

10. Pfaller MA, Moet GJ, Messer SA, Jones RN, Castanheira M. 2011. Candida bloodstream infections: comparison of species distributions and antifungal resistance patterns in community-onset and nosocomial isolates in the SENTRY Antimicrobial Surveillance Program, 2008-2009. Antimicrob Agents Chemother 55(2):561-6.

11. David L. Horn, Dionissios Neofytos, Elias J. Anaissie, Jay A. Fishman, William J. Steinbach, Ali J. Olyaei, Kieren A. Marr, Michael A. Pfaller, Chi-Hsing Chang, and Karen M. Webster. 2009. Epidemiology and Outcomes of Candidemia in 2019 Patients: Data from the Prospective Antifungal Therapy Alliance Registry. Clin Infect Dis 48:1695703

12. Luis Ostrosky-Zeichner, John H. Rex, Peter G. Pappas, Richard J. Hamill, Robert A. Larsen, Harold W. Horowitz, William G. Powderly, Newton Hyslop, Carol A. Kauffman, John Cleary, Julie E. Mangino, and Jeannette Lee. 2003. Antifungal Susceptibility Survey of 2,000 Bloodstream Candida Isolates in the United States. Antimicrob Agents Chemother 47 (10) 3149-3154

13. R. Cisterna, G. Ezpeleta, O. Telleria, J. Guinea, B. Regueiro, J. Garcia-Rodríguez, J. Esperalba, and The Spanish Candidemia Surveillance Group. 2010. Nationwide Sentinel Surveillance of Bloodstream Candida Infections in
40 Tertiary Care Hospitals in Spain. J Clin Microbiol 48 (11), 4200-4206

14. Arendrup MC, Bruun B, Christensen JJ, Fuursted K, Johansen HK, Kjaeklgaard P, Knudsen JD Kristensen L, Møller J, Nielsen L, Rosenvinge FS, Røder B, Schønheyder HC, Thomsen MK, Truberg K. 2011. National surveillance of fungemia in Denmark (2004-2009). J Clin Microbiol 49 (1), 325-34

15. Ralf Fleck, Annebärbel Dietz and Herbert Hof. 2007. In vitro susceptibility of Candida species to five antifungal agents in a German university hospital assessed by the reference brothmicrodilution method and Etest. J Antimicrob Chemother 59, 767-771

16. Borg-von Zepelin M, Kunz L, Rüchel R, Reichard U, Weig M, Gross U. 2007. Epidemiology and antifungal susceptibilities of Candida spp. to six antifungal agents: results from a surveillance study on fungaemia in Germany from July 2004 to August 2005. J Antimicrob Chemother 60 (2):424-8

17. M27-A3, Reference Method for Broth Dilution Antifungal Susceptibility Testing of Yeasts; Approved Standard Third Edition 2008, Clinical and Laboratory Standards Institute, Wayne, USA

18. M44A2, Method for Antifungal Disk Diffusion Susceptibility Testing of Yeasts; Approved Guideline-Second Edition 2009, Clinical and Laboratory Standards Institute, Wayne, USA

19. Subcommittee on Antifungal Susceptibility Testing (AFST) of the ESCMID European Committee for Antimicrobial Susceptibility Testing (EUCAST). 2008. EUCAST Definitive Document EDef 7.1: method for the determination of broth dilution MICs of antifungal agents for fermentative yeasts. Clin Microbiol Infect 14: 398-405

20. Manuel Cuenca-Estrella, Wendy Lee-Yang, Meral A. Ciblak, Beth A. Arthington-Skaggs Emilia Mellado, David W. Warnock, and Juan L. Rodriguez-Tudela. 2002. Comparative Evaluation of NCCLS M27-A and EUCAST Broth Microdilution Procedures for Antifungal Susceptibility Testing of Candida Species. Antimicrob Agents Chemother 46 (11): 3644-3647

21. A. Espinel-Ingroff, F. Barchiesi, M. Cuenca-Estrella, M. A. Pfaller, M. Rinaldi, J. L. Rodriguez-Tudela, and P. E. Verweij. 2005. International and Multicenter Comparison of EUCAST and CLSI M27-A2 Broth Microdilution Methods for Testing Susceptibilities of Candida spp. to Fluconazole, Itraconazole, Posaconazole, and Voriconazole. J Clin Microbiol 43 (8): 3884-3889

22. Maiken Cavling Arendrup, Guillermo Garcia-Effron, Cornelia Lass-Flörl, Alicia Gomez Lopez, Juan-Luis Rodriguez-Tudela, Manuel Cuenca-Estrella, and David S. Perlin. 2010. Echinocandin Susceptibility Testing of Candida Species: Comparison of EUCAST EDef 7.1, CLSI M27A3, Etest, Disk Diffusion, and Agar Dilution Methods with RPMI and IsoSensitest Media. Antimicrob Agents Chemother 54 (1): 426-439

23. M. A. Pfaller, M. Castanheira, D. J. Diekema, S. A. Messer, G. J. Moet, and R. N. Jones. 2010. Comparison of European Committee on Antimicrobial Susceptibility Testing (EUCAST) and Etest Methods with the CLSI Broth Microdilution Method for Echinocandin Susceptibility Testing of Candida Species. J Clin Microbiol 48 (5): 1592-1599

24. Manuel Cuenca-Estrella, Alicia Gomez-Lopez, Ana Alastruey-Izquierdo, Leticia Bernal-Martinez, Isabel Cuesta, Maria J. Buitrago, and Juan L. Rodriguez-Tudela. 2010. Comparison of the VITEK 2 Antifungal Susceptibility System with the CLSI and the EUCAST Broth Microdilution Reference Methods and with the Sensititre Yeast-One and the Etest Techniques for the Detection in Vitro of Antifungal Resistance in Yeasts. J Clin Microbiol 48: 1782 - 1786.

25. Maiken Cavling Arendrup, Gunnar Kahlmeter, Juan Luis Rodriguez-Tudela, and J. Peter Donnelly. 2009. Breakpoints for Susceptibility Testing Should Not Divide WildType Distributions of Important Target Species. Antimicrobial Agents Chemother 53(4): 1628-1629 
26. Morschhäuser J (2002) The genetic basis of fluconazole resistance development in Candida albicans. Biochim Biophys Acta 1587: 240-248.

27. Park S, Kelly R, Kahn JN , J. Robles, M.-J. Hsu, E. Register, W. Li, V. Vyas, H. Fan, G. Abruzzo, A. Flattery, C. Gill, G. Chrebet, S. A. Parent, M. Kurtz, H. Teppler, C. M. Douglas, and D. S. Perlin. 2005. Specific substitutions in the echinocandin target Fks1p account for reduced susceptibility of rare laboratory and clinical Candida sp. isolates. Antimicrob Agents Chemother 49 (8): 3264-73.

28. Balashov SV, Park S, Perlin DS. 2006. Assessing resistance to the echinocandin antifungal drug caspofungin in Candi$\mathrm{da}$ albicans by profiling mutations in FKS1. Antimicrob Agents Chemother 50: 2058-63.

29. Manjunath P. Pai, 1 Robin S. Turpin,2,3 and Kevin W. Garey. 2007. Association of Fluconazole Area under the Concentration-Time Curve/MIC and Dose/MIC Ratios with Mortality in Nonneutropenic Patients with Candidemia. Antimicrob Agents Chemother 51 (1): 35-39

30. Hernandez S, Lopez-Ribot JL, Najvar LK, Dora I. McCarthy, Rosie Bocanegra, and John R. Graybill. 2004. Caspofungin resistance in Candida albicans: correlating clinical outcome with laboratorysusceptibility testing of three isogenic isolates serially obtainedfrom a patient with progressive Candida esophagitis. Antimicrob Agents Chemother 48: 1382-3.

31. Katiyar S, Pfaller M, Edlind T. 2006. Candida albicans and Candida glabrata clinical isolates exhibiting reduced echinocandin susceptibility. Antimicrob Agents Chemother 50: 2892-4.

32. Laverdiere M, Lalonde RG, Baril JG, Donald C. Sheppard, Steven Park, and David S. Perlin. 2006. Progressive loss of echinocandin activity following prolonged use for treatment of Candida albicans oesophagitis. J Antimicrob Chemother 57: 705-8.

33. Miller CD, Lomaestro BW, Park S, David S. Perlin. 2006. Progressive esophagitis caused by Candida albicans with reduced susceptibility to caspofungin. Pharmacotherapy 26: $877-80$

34. Hakki M, Staab JF, Marr KA. 2006. Emergence of a Candida krusei isolate with reduced susceptibility to caspofungin during therapy. Antimicrob Agents Chemother 50: 2522-4.

35. Krogh-Madsen M, Arendrup MC, Heslet L, Jenny Dahl Knudsen. 2006. Amphotericin B and caspofungin resistance in Candida glabrata isolates recovered from a critically ill patient. Clin Infect Dis 42: 938-44.

36. Maiken C. Arendrup, Manuel Cuenca-Estrella, J. Peter Donnelly, Cornelia Lass-Flo“rl, Juan L. Rodriguez-Tudela. 2009.Association of Fluconazole Pharmacodynamics with Mortality in Patients with Candidemia. Antimicrob Agents Chemother 53 (6) 2704-2705
37. John W. Baddley, Luis Ostrosky-Zeichner, Sujata M. Bhavnani, David R. Andes. 2009. Authors' Reply. Antimicrob Agents Chemother 53 (6) 2705-2706

38. M. A. Pfaller, L. Boyken, R. J. Hollis, J. Kroeger, S. A. Messer, S. Tendolkar, and D. J. Diekema. 2008. In Vitro Susceptibility of Invasive Isolates of Candida spp. To Anidulafungin, Caspofungin, and Micafungin: Six Years of Global Surveillance. J Clin Microbiol 46 (1): 150-156

39. Shawn A. Messer, Gary J. Moet1 Jeffrey T. Kirby, and Ronald N. Jones. 2009. Activity of Contemporary Antifungal Agents, Including the Novel Echinocandin Anidulafungin, Tested against Candida spp., Cryptococcus spp., and Aspergillus spp.: Report from the SENTRY Antimicrobial Surveillance Program (2006 to 2007). J Clin Microbiol 47 (6): 1942-1946

40. Shawn A. Messer, Ronald N. Jones, Gary J. Moet, Jeffrey T. Kirby, and Mariana Castanheira. 2010. Potency of Anidulafungin Compared to Nine Other Antifungal Agents Tested against Candida spp., Cryptococcus spp., and Aspergillus spp.: Results from the Global SENTRY Antimicrobial Surveillance Program (2008). J Clin Microbiol 48 (8): 2984-2987

41. D. J. Diekema, S. A. Messer, L. B. Boyken, R. J. Hollis, J. Kroeger, S. Tendolkar, and M. A. Pfaller. 2009. In Vitro Activity of Seven Systemically Active Antifungal Agents against a Large Global Collection of Rare Candida Species as Determined by CLSI Broth Microdilution Methods. J Clin Microbiol 47 (10): 3170-3177

42. S. C. A. Chen, D. Marriott, E. G. Playford, Q. Nguyen, D. Ellis, W. Meyer, T. C. Sorrell, M. Slavin and the Australian Candidaemia Study. 2009. Candidaemia with uncommon Candida species: predisposing factors,outcome, antifungal susceptibility, and implications for management. Clin Microbiol Infect 15: 662-669

Received: March 7, 2011 / Accepted: March 20, 2011

Address for correspondence:

Arne C. Rodloff

Institut für Medizinische Mikrobiologie

und Infektionsepidemiologie

Universitätsklinikum Leipzig

Liebigstr. 21

04103 Leipzig

Germany

Tel. +493419715200

E-mail: acr@medizin.uni-leipzig.de 